\title{
AiMT
}

Advances in Military Technology

Vol. 15, No. 2, 2020, pp. 287-299

ISSN 1802-2308, eISSN 2533-4123

DOI 10.3849/aimt.01284

\section{Evaluation of Retina Exposition by Optical Incapacitating Device}

\author{
F. Racek ${ }^{*}$, T. Baláž ${ }^{1}$, J. Leuchter ${ }^{2}$, J. Krejčí ${ }^{1}$ and J. Bajer ${ }^{2}$ \\ ${ }^{1}$ Department of Weapon and Ammunition, University of Defence in Brno, Czech Republic \\ ${ }^{2}$ Department of Aircraft Technology, University of Defence in Brno, Czech Republic
}

\begin{abstract}
The manuscript was received on 2 January 2019 and was accepted after revision for publication as research paper on 21 August 2020.
\end{abstract}

\begin{abstract}
:
It has turned out that the usage of non-lethal weapons is an effective way of tackling adversaries in many security scenarios. The security staff's goal is to use the lowest possible level of power needed to manage successfully the situation during the conflicts with adversaries. The security staff can employ a wide spectrum of possible reactions beginning with the voice alert through the physical interaction up to the usage of e.g. firearms. The optical incapacitating device is one of possible realizations of non-lethal weapons. Therefore, the article deals with the description of the designed optical incapacitating device and the evaluation of retina exposition when used.
\end{abstract}

\section{Keywords:}

eye model, non-lethal weapon, retina, spot diagram

\section{Introduction}

Security service (independent in military forces, police, prison guards or air marshals, etc.) can generally be undertaking the mission where minimizing collateral damages overcomes the primary task. To accomplish the task, the violence-controllers require non-lethal capability to react to a potential threat. In accordance with [1], the non-lethal weapon (NLW) is "explicitly designed and primarily employed to incapacitate personnel or materiel immediately, while minimizing fatalities, permanent injury to personnel, and undesired damage to property, facilities, materiel, and the environment". Evaluation of electromagnetic radiation in area of visible wavelengths forming the optical incapacitating device (OID) is one of the possible ways of creating the NLW acting in both antipersonal and anti-material actions.

To fulfill the anti-personal security task, the OIDs affect the human vision. The bright light sources provide five levels of physical interaction with adversaries [1]:

\footnotetext{
* Corresponding author: Department of Weapon and Ammunition, University of Defence in Brno, Kounicova 156/65, CZ-662 10 Brno, Czech Republic. Phone: +420 973443 361, E-mail: frantisek.racek@unob.cz.ORCID 0000-0003-4985-2557.
} 
- language-independent, unequivocal warning,

- psychological impact such as distraction and fear,

- temporarily impaired vision,

- physiological response to the light such as disorientation and nausea,

- reduced ability to perform hostile acts such as throwing objects, attacking, or aiming firearms.

Based on the level and behavior of the bright light attack, the response of human vision splits into three main types [2]:

- glare,

- flash-blindness or afterimages,

- psychosomatic effects.

The OID is in principle a non-lethal weapon. Nonetheless, the dangerousness of the usage of such weapon lies in the possibility of permanent damage of adversary's vision. The eye-safe operation mode is defined by the hygienic standards as Maximum Permissible Exposure (MPE). The exposure is a physical quantity that depends on light energy, time, and irradiated area. The MPE varies from $0.0001 \mathrm{~W} / \mathrm{cm}^{2}$ in the case of long time exposure up to $0.0583 \mathrm{~W} / \mathrm{cm}^{2}$ in the case of extremely short exposure [3-5]. If the MPE is taken as $100 \%$ exposure, the effect on human vision can be divided into subcategories as illustrated in Fig. 1. In accordance with presumed intensity of attack, the optical non-lethal weapon should inhere with appropriate power. The main task is not to design a sufficiently powerful weapon, but to assess correctly the effects of the weapon on the vision.

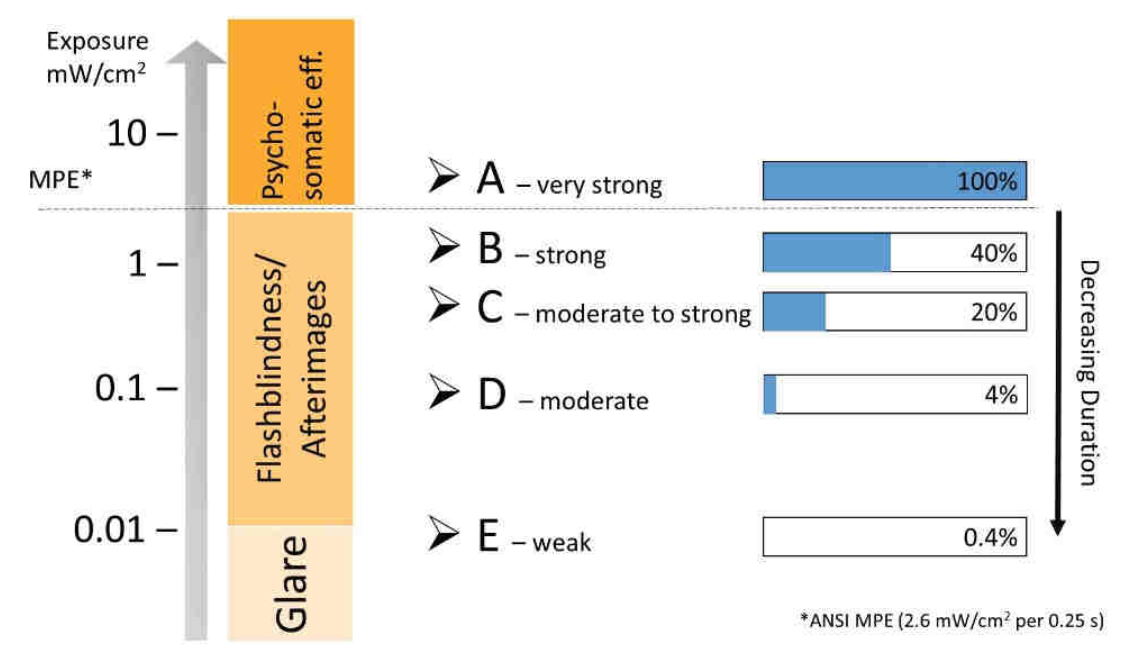

Fig. 1 The response of human vision on the bright light attack; [2] - modified

\section{The Design of OID}

Generally, the design of OID relates to a portable solid-state lighting device. The device is powered from an internal power supply unit and it is operated through micro controller. The output bright light is generated in the LED, which is controlled by both microprocessor and LED driver. The radiated light is collimated by optical elements. The simplified block diagram of the OID is presented in Fig. 2. 


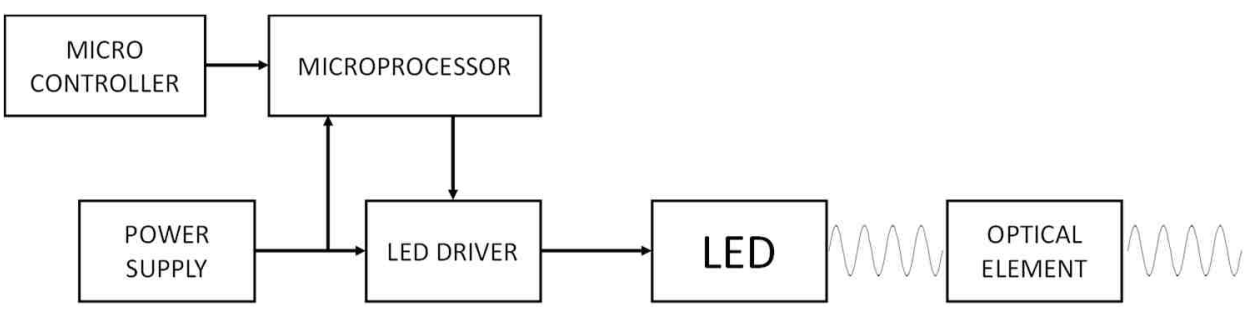

Fig. 2 The structure of Optical Incapacitating Device

As a bright light, either laser or LED can be used. By using a laser, we can easily achieve the desired incapacitating effect. Moreover, special diffuse elements have to be used in optical system design to achieve the safety limits for consumable laser device. On the other hand, the usage of a laser in such a device is, besides the construction difficulties, connected with a set of safety and hygienic standards and an administrative burden. LED-based OID providing sufficient level of illumination requires a significant increase in power to achieve an acceptable incapacitating effect. Nowadays, advances in LEDs, electronics and power sources allow creation of portable LED-based OID. The electronics control unit receives power from the power supply unit and controls the emission of light from the LED to achieve desired light level and mode. The function of optical element system is to form a desired light beam from the LED. Since the LED emits light into a high divergence angle, the optical system of OID primary function is to form a light beam with an appropriate divergence angle and energy distribution. The optical system of OID is a non-imaging collimating optical systems that can be successfully created by reflector of appropriate surface shape only. The usage of such an optical system contributes to an increase in irradiance produced by the OID. Application of the mentioned reflector systems does not increase the overall diameter of the apparatus and, at the same time, it reduces the complexity of the OID. Both the level and the distribution of light energy in the eye entrance pupil plane has to be known to evaluate the eye retina exposition. As mentioned above, both level and energy distributions are determined by the optical system. The optical system used for modeling the eye retina exposition is a simple conical reflector, as described in Fig. 3. The conical angle of the reflector is $2 \sigma$, with the minimal diameter $D_{0}$, the maximal diameter $D$ and the length of reflector $h$. The LED is situated in the origin of coordinate system. This lies on optical system axis in the distance $v$ from the vertex of the cone. The result of the modeled irradiation in the plane of analysis is illustrated in Fig. 5.

\section{Eye Retina Exposition Model}

As mentioned in the previous paragraph, the eye-safe operation mode of OID is defined by MPE, as it is stated in the hygiene standards. The value of MPE defines the maximal value of radiation entering the eye. Since the MPE is determined for the most unfavorable condition (maximal eye pupil diameter and scotopic vision), it is ensured that the eye will not be damaged under any circumstances. Unfortunately, this methodology allows the low exposition of the eye retina in some cases. Thus the incapacitating performance of OID may not be fulfilled, although the MPE is reached on eye pupil plane.

Hence, we designed a model of eye retina exposition to simulate and determine the real level of retina exposition. The advantages of the analysis using the developed model 
are illustrated in Fig. 5. The model of OID (Fig. 3) creates a certain distribution of irradiation in the plane of eye pupil (Fig. 4). Using the current methodology, the MPE should be performed. The introduced model allows to do an in-depth analysis. The precise model of the eye produces the actual distribution of eye retina irradiation as an output of eye pupil irradiation. The energy distribution on eye retina permits the determination of both damage threshold and incapacitating threshold as a function of current circumstances.

Nowadays, the following five eye models are the most commonly eye models used for theoretical eye imaging properties: Helmholtz-Laurance [6], Gullstrand [6], Emsley [7], Greivenkamp [6] and Liou \& Brennan [7]. According to our research, the most highly valued model is the Liou \& Brennan model, containing some features of the biological eye, not considered in other models. These include, for example, the distribution of the refractive index gradient and pupil's decentralization. This model also exhibits a high compliance with biological eye, and it has a great reliability, because it takes into account the mean value of empirical measurements of the eye in vivo [5]. A detailed analysis of the image quality between the eye models showed that the first four schematic eyes had a better image quality than expected in a normal healthy ametropic eye in vivo. The Liou \& Brennan eye model is assumed now as the most closely related to their biological properties in vivo.

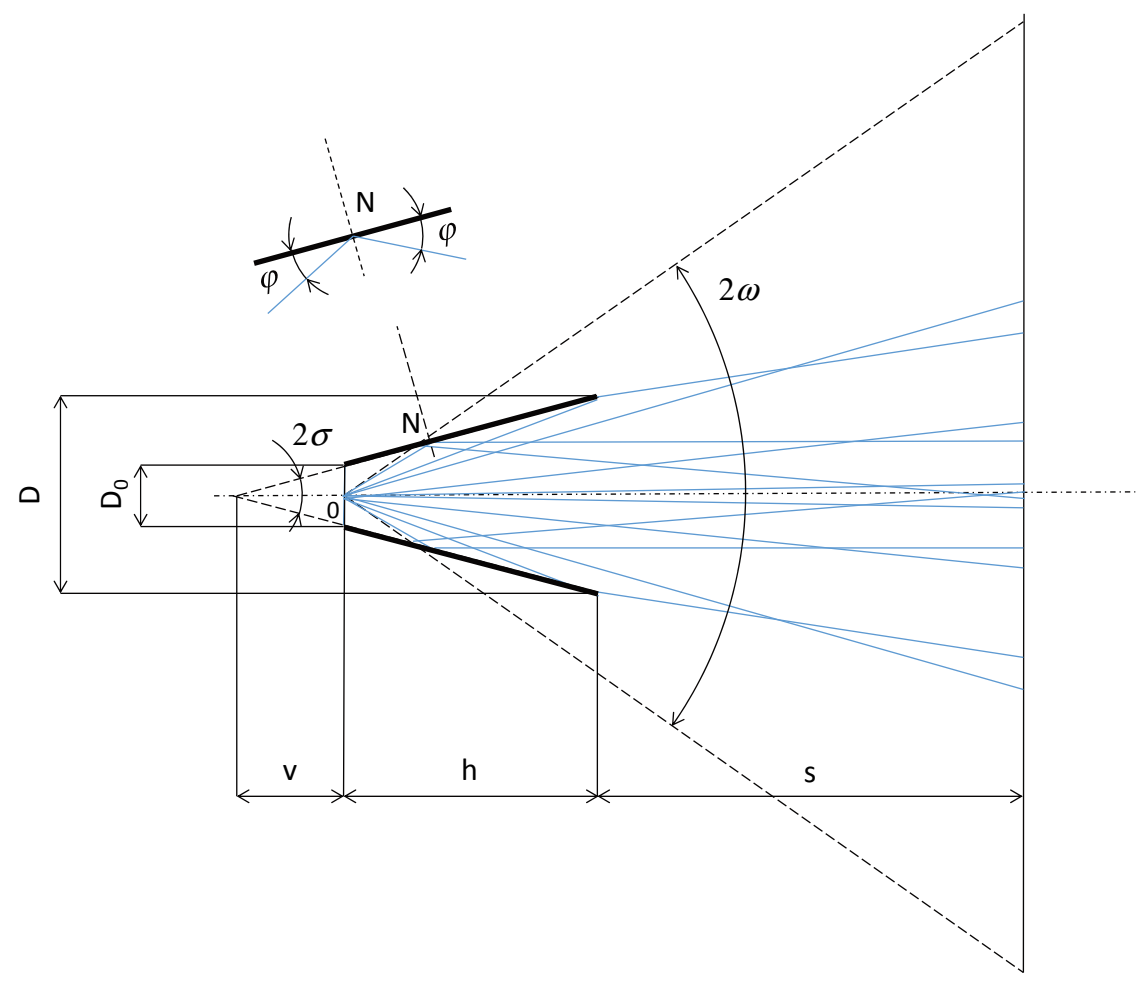

Fig. 3 OID optical system used for modeling of the eye retina exposition 


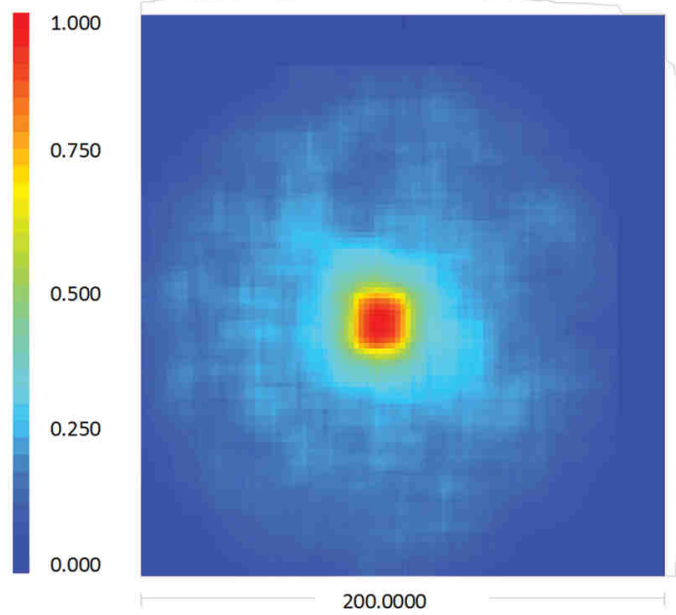

Target surface: 3

Rays: 7762

Pixel combined: 10

Power: 2.97800 (W)

TRA no

POL no

FWHM-X: 0.0000

FWHM-Y: 1.9802

Fig. 4 Distribution of normalized light energy irradiation in the plane of analysis

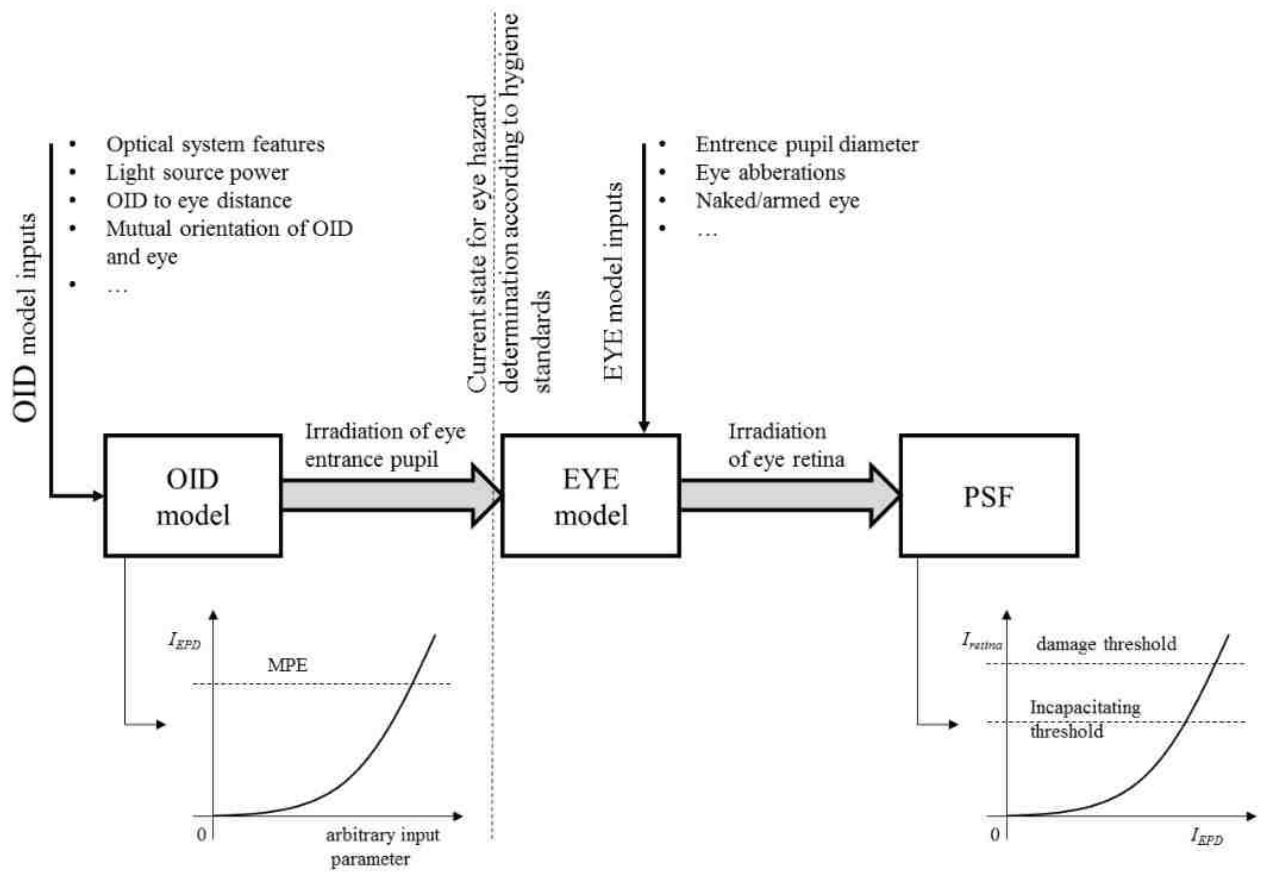

Fig. 5 The structure of the model of eye retina exposition

The analysis of the eye model and the simulation of the intensity distribution over the retina were realized using the Optalix software package [8]. This software is widely used for analysis and synthesis of optical systems.

Liou \& Brennan's eye model presented in [7] is characterized by considering variation in lens refractive index gradient, taking into account the pupil's decentralization 
and tilt, which is present for the case of $4 \mathrm{~mm}$ entrance pupil diameter (EPD) of the biological eye. Our model was designed for variable EPD, and the eye- to- object distance of approximately $44 \mathrm{~cm}$. Other aperture values would definitely alter the shape and refractive index of the ophthalmic lens. When analyzing the distribution of retinal illumination by a spot source, we used two characteristics, the spot diagrams and the point spread function (PSF).

The spot diagram is a two-dimensional graph of distribution of the ray's intersection from the object point in the image plane. The spot diagram represents the result of imaging of all off-axis rays incident on the "square grid" located in the entrance pupil of the system, passing through the optical system and reaching on the image plane. For the optical system with a smaller aperture, the diffraction effects can cause more significant image un-sharpness than caused by the geometric aberration. The light passing through the small circular aperture forms the diffractive pattern consisting of both a bright central circle and low contrast peripheral circles. The bright central circle is known as the Airy disk. Equally, when the eye focuses on a dotted object, the image created on the retina will be a circle with an Airy disk size, even in an ideal case when we do not consider geometric aberrations.

Wave-front aberration is a limiting factor in image quality for large apertures, and diffraction sets the threshold for small apertures. The design of spot diagrams is one of the methods of visualization of aberration effects and it predetermines image quality. The distribution of the intersection of the rays depends on the optical system properties. For our analysis, a sample of a square grid in the EPD was selected for ray tracing, where the main ray is situated in the middle.

The value of the root mean square (RMS) radius, also called the Gaussian moment, is another figure of merit that relies on the Spot Diagram. It is calculated as the RMS of all distances between each marginal intersection $\left(x_{i} ; y_{i}\right)$ and the reference point $\left(x_{0} ; y_{0}\right)$ generated by the main ray intersection. The RMS radius is given by:

$$
r_{\mathrm{rms}}=\frac{1}{n} \sum_{i=1}^{n} \sqrt{\left(x_{i}-x_{0}\right)^{2}+\left(y_{i}-y_{0}\right)^{2}}
$$

where $n$ is the total number of assumed rays.

The distribution of the amplitude $A(x, y)$ in the exit pupil plane and the corresponding wavelength aberration $W(x, y)$ defines the complex pupil function $P(x, y)$. The normalized coordinates in the exit pupil plane are $(x, y)$

$$
P(x, y)=f(x)=\left\{\begin{array}{cc}
A(x, y)=\exp \frac{2 \pi j W(x, y)}{\lambda} & \text { for } x^{2}+y^{2} \leq \frac{D_{\mathrm{o}}^{2}}{4} \\
0 & \text { for } x^{2}+y^{2}>\frac{D_{\mathrm{o}}^{2}}{4}
\end{array}\right.
$$

where $j$ is the imaginary unit and $D_{\mathrm{o}}$ is eye pupil diameter. The intensity distribution $I(x, y)$ in the exit pupil plane is given

$$
I(x . y)=[A(x, y)]^{2}
$$

Diffraction irradiation (illumination) $|h(u, v)|^{2}$ of the point light source in the image plane with the coordinates $(u, v)$ is well approximated by the function: 


$$
|h(u, v)|^{2}=\frac{\left[\int_{-\infty}^{+\infty} \int_{-\infty}^{+\infty} P(x, y) \mathrm{e}^{-2 \pi \mathrm{j}(x u+y v)} \mathrm{d} x \mathrm{~d} y\right]^{2}}{\left[\int_{-\infty}^{+\infty} \int_{-\infty}^{+\infty} P(x, y) \mathrm{d} x \mathrm{~d} y\right]^{2}}
$$

The PSF describes the intensity of the diffraction pattern of a point located in the object plane created by the optical system. The PSF is calculated from the waveform in the plane of the output pupil of the optical system using the double Fourier integral (4). The aperture properties and uneven illumination due to the apodization are taken into account. The more compact spot diagrams indicate smaller aberrations.

The optical parameters of used Liou \& Brennan eye model and its optical diagram are presented in Tab. 1 and Fig. 6, respectively. The spot diagrams of the Liou-Brennan eye model are shown in the Figs 7-10. The spot light source as an object is displayed depending on the wavelength $\lambda=\{510,520,532,540,550\}[\mu \mathrm{m}]$ and the eye field of view $\{0,5,10,15\}$. The EPD ranges inside the interval of $2-8 \mathrm{~mm}$. It is easy to find out from the spot diagrams, that the Liou \& Brennan model gives the best quality for a $4 \mathrm{~mm}$ EPD.

When calculating the distribution of retinal illumination, we assumed that:

- the center of the pupil of the eye lies on the axis of the OID radiation,

- the intensive spot light source emits a constant luminous flux and

- there is no other light source in the eye field of view.

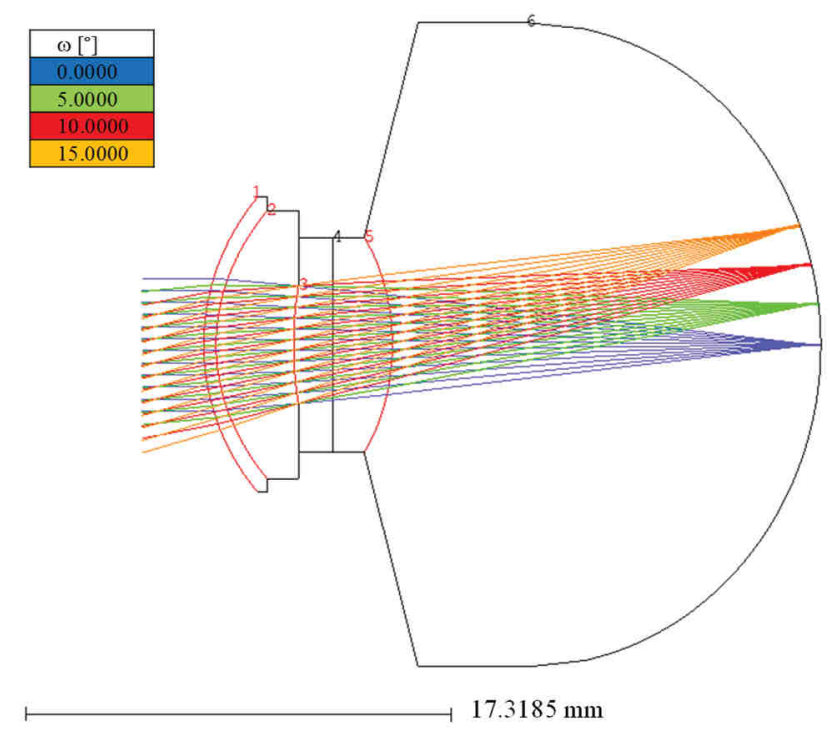

Fig. 6 Optical diagram of Liou \& Brennan eye model (notice: the colors of the rays represent different incident angle, not the light spectrum)

The coefficient of eye transmittance for the LED wavelength was determined using the spectral transmission curves at several depth locations inside the eye [9]. The exposure time for the retinal illumination is limited by the physiological response, i.e. until 
the eyelid closes, that is approximately $0.25 \mathrm{~s}$. Assuming the value of EPD and the expected distance from the spot light source, we could consider the level of illumination in the plane of the cornea to be constant.

Tab. 1 Parameters of Liou \& Brennan eye model

\begin{tabular}{|c|c|c|c|c|c|l|}
\hline \#surface & type & $\boldsymbol{r}[\mathbf{m m}]$ & $\boldsymbol{d}[\mathbf{m m}]$ & Material & $\boldsymbol{h}[\mathbf{m m}]$ & \multicolumn{1}{|c|}{ Note } \\
\hline Object & S & $\infty$ & $\infty$ & & 0 & \\
\hline 1 & A & 7.77 & 0.50 & CORNEA & 5.50 & cornea, outer \\
\hline 2 & A & 6.4 & 3.16 & AQUEOUS & 5.00 & cornea, inner \\
\hline $3(\mathrm{AC})$ & AI & 12.4 & 1.59 & GRIN & 2.21 & iris + lens, $1^{\text {st }}$ \\
\hline 4 & SI & $\infty$ & 2.34 & GRIN & 4.00 & \\
\hline 5 & A & -8.1 & 17.40 & VITREOUS & 4.00 & ${\text { crystalline lens, } 1^{\text {st }}}^{\text {nd }}$ \\
\hline 6 (Image) & S & -12.0 & 1.21 & & 12.00 & crystalline lens, $^{\text {nd }}$ \\
\hline
\end{tabular}

\section{Analysis}

OID due to its radiation characteristics (see Figs 3 and 4) can be considered as a spot light source. In this case, the PFS approximates very well the distribution of light intensity on the retina of the eye.

Applying simpler models of the eye than the Lui-Brennan model to the analysis of retina illumination, we can only parametrically change the eye pupil diameter $D_{\text {o }} \in\langle 2 ; 8\rangle \mathrm{mm}$. The retina illumination level will only depend on the diameter $D_{\text {o }}$ for a constant luminous source. Thus, the ratio between the energy entering the eye of the smallest and the largest pupil will be 16. However, in the case of Lui-Brennan model of the eye, the aberration state of the optical system changes significantly as the pupil diameter changes. Further, the model allows varying of the immediate accommodation of the optical system of the eye. In the following figures (Figs 7-10), we considered that the eye is accommodated to infinity.

In addition to the numerical calculation of PSF according to Eq. (4), we used a geometric approximation in the form of a SPOT diagram to analyze the illumination of the retina. The SPOT diagram is a scattering pattern formed by intersections of individual beams with the retina surface. We gradually placed the OID light source at different angular distances from the optical axis $\sigma=\left\{0^{\circ}, 5^{\circ}, 10^{\circ}, 15^{\circ}\right\}$.

Comparing the areas of the SPOT diagrams, we can formulate following formulate/produce results. The amount of the energy entering the eye in the case of the pupil diameter $D_{\mathrm{o}}=6 \mathrm{~mm}$ is nine times greater compared with the pupil diameter $D_{\mathrm{o}}=2 \mathrm{~mm}$. On the other hand, the scattering pattern of the SPOT diagram for $D_{\mathrm{o}}=2 \mathrm{~mm}$ is more than nine times larger. This implies that local light exposure on the retina at pupil diameter $D_{\mathrm{o}}=6 \mathrm{~mm}$ will be considerably less. The decrease in exposure is caused by aberration effects. Even more, these differences become more significant for increasing angular distance of the light source from the optical axis.

The result of the analysis is shown in Tab. 2 which summarizes the resulting radii of SPOT diagrams of all cases displayed in Figs 7-10 for different wavelengths and angular distances. For angular distances, the mean $\overline{r_{\mathrm{m}}}$ over all wavelengths has been computed. Since the amount of energy falling on the retina varies with the ratio of EPD squares, we can compute normalized retina irradiation as a function of eye aberration state. The resulting normalized irradiation $E_{\mathrm{n}}$ is also displayed in Tab. 2. 

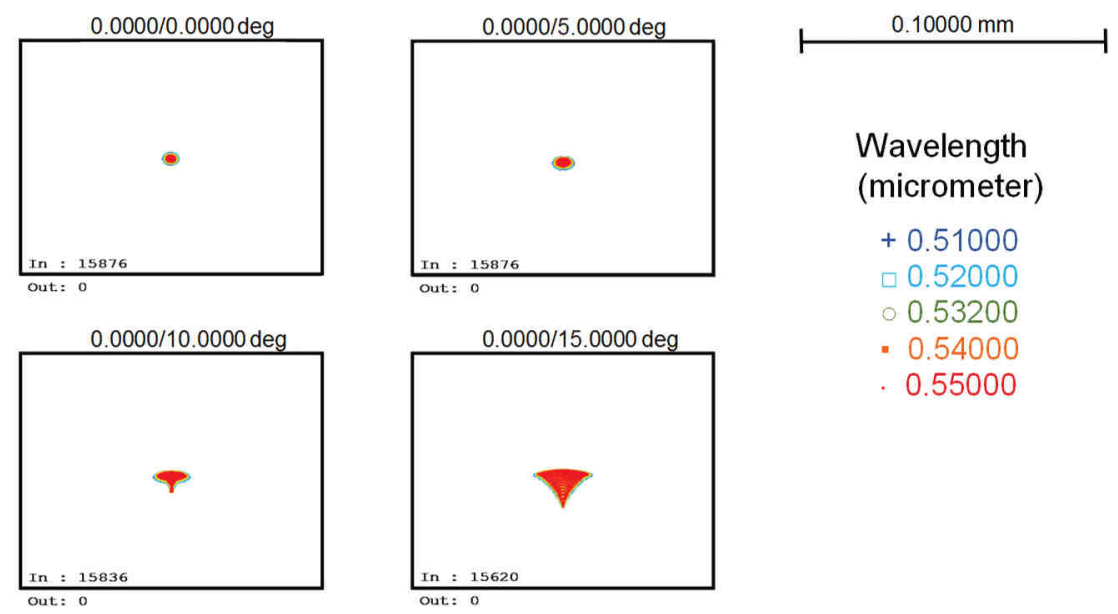

Fig. 7 Spot diagrams of Liou \& Brennan eye model for $2 \mathrm{~mm}$ EPD and different angular distance $\sigma$ of OID from optical axis, upper left $\sigma=0^{\circ}$, upper right $\sigma=5^{\circ}$, lower left $\sigma=10^{\circ}$ and lower right $\sigma=15^{\circ}$

\section{Conclusion}

The optical incapacitating device is a non-lethal weapon influencing the human vision by the lowest possible level of light energy needed to achieve successfully the predominance during the conflicts. The paper described the design of such device and the model of retina exposition in its use.

In addition, the article presents a model that was created based on the need to determine in detail the level of illumination (irradiation) of the retina of the eye caused by OID. To simulate and plan the tactical use of OID in real conditions, it is necessary to model and determine the illumination of the retina of the eye under different lighting conditions, altered eye adaptation to the ambient light situation, and the possibility of using prescription or protective glasses.

This issue is usually described in the literature, in the case of a simple model of the human eye, which does not reflect, for example, the aberration state of the optical system of the eye. Retinal illumination is commonly described by means of fundamental paraxial relations. The ordinary simple models are not sufficient when we consider the use of OID on the edge of maximum permissible exposure (MPE). The MPE defined by hygiene standards is specified for the "worst" possible conditions, i.e. the eye is adopted for night conditions and wider eye pupil diameter. Under the real conditions, the OID must fulfill its functionality even at daylight level, i.e. the MPE level must be exceeded. In these cases, it is necessary to use models of the human eye that simulate the real properties of the optical system of the eye and to further consider empirical practice to simulate the distribution of the retina illumination. 
Tab. 2 Radii $r_{\mathrm{ms}}$ of SPOT diagrams in tangential plane for different angular distances from the optical axis $0^{\circ}$ to $15^{\circ}$

\begin{tabular}{|c|c|c|c|c|c|}
\hline \multirow{2}{*}{$\begin{array}{c}D_{\mathbf{0}} \\
{[\mathrm{mm}]}\end{array}$} & \multirow{2}{*}{$\begin{array}{c}\lambda \\
{[\mu \mathrm{m}]}\end{array}$} & \multicolumn{4}{|c|}{$r_{\mathrm{ms}}[\mathrm{mm}]$} \\
\hline & & $\mathbf{0}^{\circ}$ & $5^{\circ}$ & $10^{\circ}$ & $15^{\circ}$ \\
\hline \multirow{7}{*}{2} & 0.550 & 0.001380 & 0.00150 & 0.00200 & 0.00360 \\
\hline & 0.540 & 0.001275 & 0.00138 & 0.00194 & 0.00355 \\
\hline & 0.532 & 0.001120 & 0.00124 & 0.00190 & 0.00350 \\
\hline & 0.520 & 0.000950 & 0.00118 & 0.00880 & 0.00345 \\
\hline & 0.510 & 0.000800 & 0.00110 & 0.00185 & 0.00340 \\
\hline & $\overline{r_{\mathrm{m}}}[\mathrm{mm}]$ & 0.001105 & 0.001280 & 0.001914 & 0.003500 \\
\hline & $E_{\mathrm{n}}[-]$ & 1 & 0.745 & 0.333 & 0.1 \\
\hline \multirow{7}{*}{4} & 0.550 & 0.0042 & 0.00500 & 0.00720 & 0.0104 \\
\hline & 0.540 & 0.0040 & 0.00480 & 0.00700 & 0.0102 \\
\hline & 0.532 & 0.0037 & 0.00465 & 0.00680 & 0.0100 \\
\hline & 0.520 & 0.0034 & 0.00450 & 0.00640 & 0.0098 \\
\hline & 0.510 & 0.0032 & 0.00430 & 0.00625 & 0.0096 \\
\hline & $\overline{r_{\mathrm{m}}}[\mathrm{mm}]$ & 0.0037 & 0.00465 & 0.00673 & 0.01 \\
\hline & $E_{\mathrm{n}}[-]$ & 0.357 & 0.226 & 0.1 & $\begin{array}{l}0.0488 \\
\end{array}$ \\
\hline \multirow{7}{*}{5} & 0.550 & 0.01000 & 0.0110 & 0.0130 & 0.0175 \\
\hline & 0.540 & 0.00950 & 0.0105 & 0.0128 & 0.0170 \\
\hline & 0.532 & 0.00900 & 0.0097 & 0.0127 & 0.0168 \\
\hline & 0.520 & 0.00885 & 0.0095 & 0.0126 & 0.0165 \\
\hline & 0.510 & 0.00875 & 0.0090 & 0.0125 & 0.0160 \\
\hline & $\overline{r_{\mathrm{m}}}[\mathrm{mm}]$ & 0.00922 & 0.00994 & 0.01272 & 0.01676 \\
\hline & $E_{\mathrm{n}}[-]$ & 0.09 & 0.07700 & 0.047 & 0.027 \\
\hline \multirow{7}{*}{6} & 0.550 & 0.0200 & 0.02100 & 0.0240 & 0.0287 \\
\hline & 0.540 & 0.0195 & 0.01980 & 0.0237 & 0.0283 \\
\hline & 0.532 & 0.0192 & 0.01970 & 0.0235 & 0.0280 \\
\hline & 0.520 & 0.0190 & 0.01960 & 0.0233 & 0.0275 \\
\hline & 0.510 & 0.0185 & 0.01930 & 0.0230 & 0.0270 \\
\hline & $\overline{r_{\mathrm{m}}}[\mathrm{mm}]$ & 0.01924 & 0.01988 & 0.0235 & 0.0279 \\
\hline & $E_{\mathrm{n}}[-]$ & 0.03 & 0.02 & 0.02 & $\mathbf{0}$ \\
\hline
\end{tabular}

\section{Acknowledgement}

This research was supported by the Ministry of Interior of the Czech Republic, project VI20172020058 Ballistic protection means and incapacitating device for Czech Police Air-marshals. 

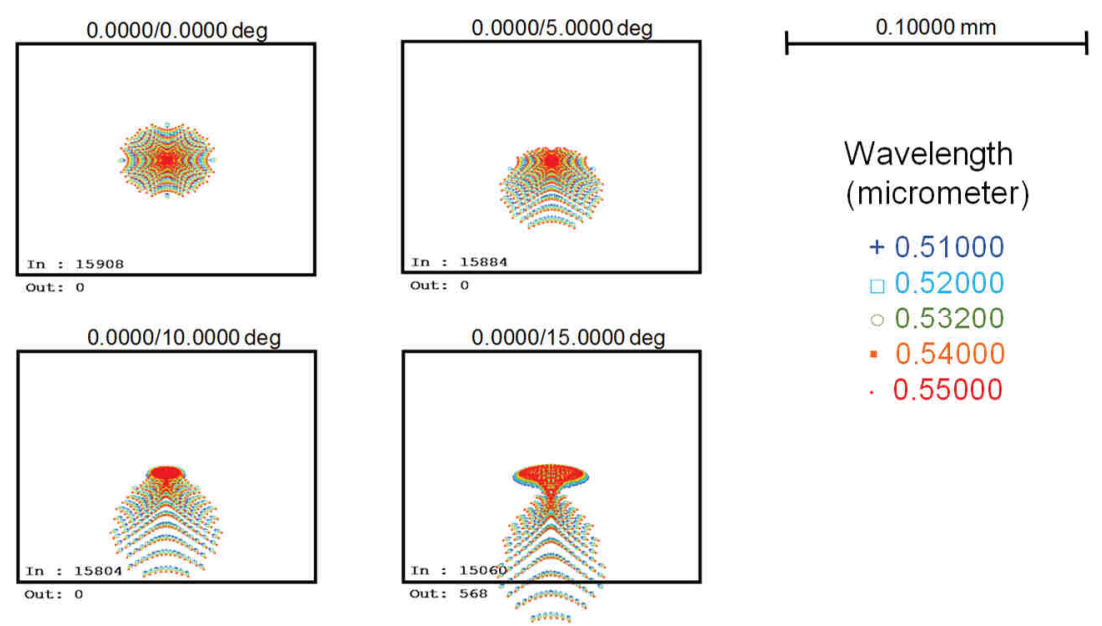

Wavelength

(micrometer)

$+0.51000$

$\square 0.52000$

0.53200

- 0.54000

. 0.55000

Fig. 8 Spot diagrams of Liou \& Brennan eye model for $4 \mathrm{~mm}$ EPD and different angular distance $\sigma$ of OID from optical axis, upper left $\sigma=0^{\circ}$, upper right $\sigma=5^{\circ}$, lower left $\sigma=10^{\circ}$ and lower right $\sigma=15^{\circ}$
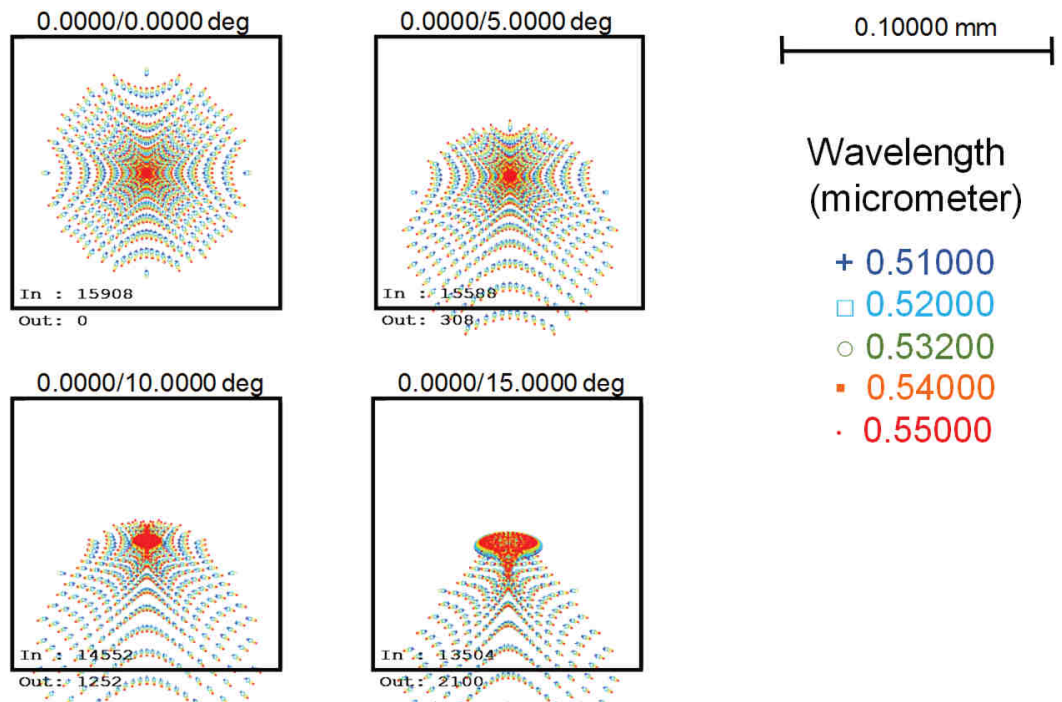

Wavelength (micrometer)

$+0.51000$

$\square 0.52000$

0.53200

- 0.54000

. 0.55000

Fig. 9 Spot diagrams of Liou \& Brennan eye model for $5 \mathrm{~mm}$ EPD and different angular distance $\sigma$ of OID from optical axis, upper left $\sigma=0^{\circ}$, upper right $\sigma=5^{\circ}$, lower left $\sigma=10^{\circ}$ and lower right $\sigma=15^{\circ}$ 


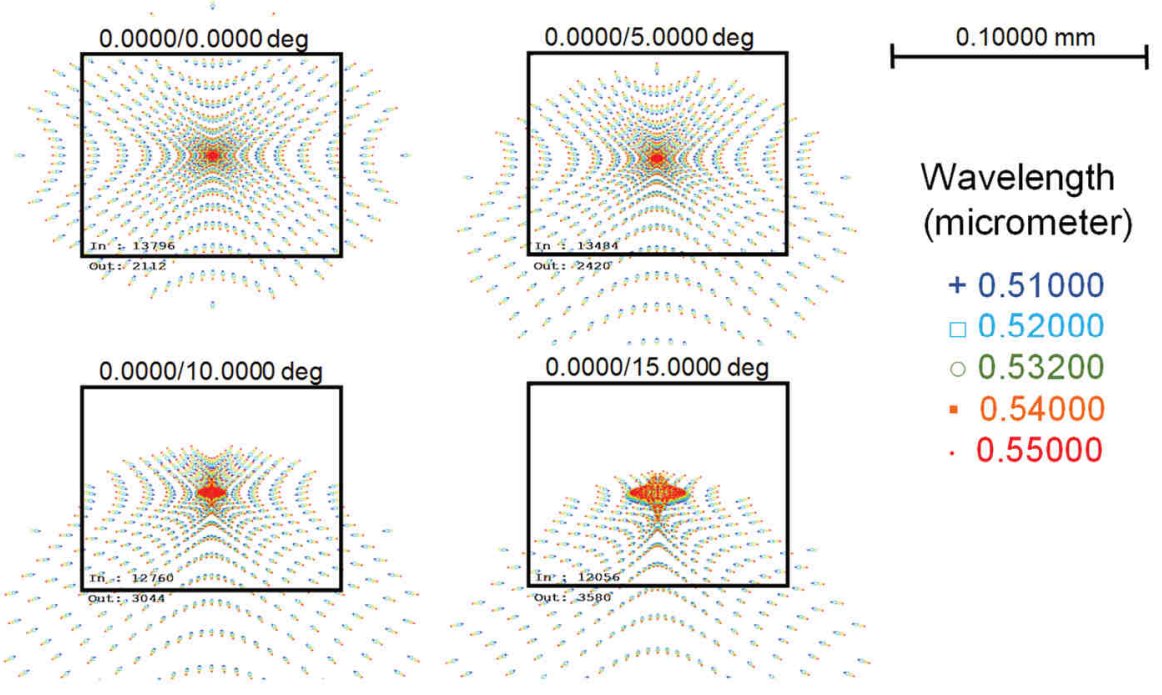

Fig. 10 Spot diagrams of Liou \& Brennan eye model for $6 \mathrm{~mm}$ EPD and different angular distance $\sigma$ of OID from optical axis, upper left $\sigma=0^{\circ}$, upper right $\sigma=5^{\circ}$, lower left $\sigma=10^{\circ}$ and lower right $\sigma=15^{\circ}$

\section{References}

[1] TOCCI, N.C., TOCCI, M.D., GERMAN, J.D. and CRAMER, E.C. Enhanced NonLethal Visual Security Device. US Patent US6190022, 1995. 17 p.

[2] RUBTSOV, V. LED-based Incapacitating Apparatus and Method. US Patent US7500763, 2005. $30 \mathrm{p}$.

[3] WOODS, M., BROWN, M., DABNEY M., KING, J. and TONCHEVA, G. Evaluation of ANSI Z136.1-2014 and Comparison with Z136.1-2007 and Z136.8-2012 [online]. In Proceedings of the International Laser Safety Conference. Albuquerque, 2015, 1-10. [viewed 2019-06-09]. Available from: https://www.osti.gov/ servlets/purl/1244847

[4] ANSI Z136.1-2007 [online]. American National Standard for Safe Use of Lasers [viewed 2019-06-07]. Available from: https://loci.wisc.edu/sites/default/files/201704/American\%20National\%20Standard\%20for\%20Safe\%20Use\%20of\%20Lasers.pdf

[5] ALMEIDA, M.S. and CARVALHO, L.A. Different Schematic Eyes and their Accuracy to the in vivo Eye: A Quantitative Comparison Study [online]. Brazilian Journal of Physics, 2007, vol. 37, no. 2A, p. 378-387. DOI 10.1590/S010397332007000300008.

[6] GOBBI, P.G. Modeling the Optical and Visual Performance of the Human Eye. Bellingham: SPIE Press, 2013. 430 p. ISBN 978-0-81-949254-8.

[7] LIOU, H.L. and BRENNAN, N.A. Anatomically Accurate, Finite Model Eye for Optical Modeling. Journal of the Optical Society of America A. 1997, vol. 14, no. 8, p. 1684-1695. DOI 10.1364/JOSAA.14.001684. 
[8] OPTALIX. Software for Optical Design, Thin-Films and Illumination. Version 10.25 [online]. 2019. [viewed 2019-05-09]. Available from: www.optenso.com

[9] GROSS, H., BLECHINGER, F. and ACHTNER, B. ed. Handbook of Optical Systems, Volume 4: Survey of Optical Instruments. Weinheim: Wiley, 2015. 1092 p. ISBN 978-3-527-69924-7. 